\title{
EDAD MATERNA AVANZADA Y MORBILIDAD OBSTÉTRICA
}

\author{
Félix Dasio Ayala Peralta1 ${ }^{1}$ Enrique Guevara Ríos², Miguel Angel Rodriguez Herrera³, \\ Richard Ayala Palomino ${ }^{4}$, Luis Alberto Quiñones Vásquez ${ }^{5}$, Dasio Ayala Moreno ${ }^{6}$, \\ Antonio Mambret Luna Figueroa ${ }^{7}$, César Carranza Asmat ${ }^{8}$, Jovanna Ochante Rementería ${ }^{9}$
}

\begin{abstract}
RESUMEN
Objetivo: Evaluar el resultado obstétrico de gestantes con edad materna avanzada (EMA) atendidas en el Instituto Nacional Materno Perinatal. Materiales y métodos: Estudio observacional, descriptivo y retrospectivo de 321 egresos maternos ocurridas durante el periodo de 1 de enero al 30 de junio 2016. Resultados: Edad promedio 38,8 $\pm 3,4$ años. Conviviente en $63,9 \%$, secundaria completa $68,3 \%$, ama de casa $80,4 \%$ y estado social bajo $66,5 \%$. Sin control prenatal en $31 \%$. Las principales comorbilidades obstétricas fueron: preeclampsia, parto pretérmino $(p<0,001)$, ruptura prematura de membranas, desgarro perineal I grado, anemia moderada/severa e infección urinaria. La razón de muerte materna (RMM) fue 4,56 x 100000 y la principal causa de defunción materna fue la hemorragia postparto. Conclusión: Las gestantes $\geq 35$ años de edad presentan una mayor incidencia de cesárea, parto pretérmino, ruptura prematura de membranas, anemia moderada/severa y la preeclampsia.
\end{abstract}

Palabras clave: Edad materna avanzada; Patología obstétrica; Embarazo de alto riesgo (Fuente DeCS BIREME).

\section{ADVANCED MATERNAL AGE AND OBSTETRIC MORBIDITY}

\begin{abstract}
Objective: To evaluate the obstetric outcome of pregnant women with advanced maternal age (EMA) treated at the National Maternal Perinatal Institute. Materials and methods: Observational, descriptive and retrospective study of 321 maternal outgoings occurred during the period from January 1 to June 30, 2016. Results: Average age 38,8 $\pm 3,4$ years. Living in $63.9 \%$, high school $68,3 \%$, housewife $80,4 \%$ and social status under $66,5 \%$. Without prenatal control in $31 \%$. The main obstetric comorbidities were: preeclampsia, preterm delivery $(p<0,001)$, premature rupture of membranes, perineal tears I grade, moderate / severe anemia and urinary infection. The maternal death rate (MMR) was 4,56 100000 and the main cause of maternal death was postpartum haemorrhage. Conclusion: Pregnant women $\geq 35$ years of age have a higher incidence of cesarean section, preterm delivery, premature rupture of membranes, moderate / severe anemia and preeclampsia.
\end{abstract}

Key words: Advanced maternal age; Obstetric pathology; High risk pregnancy (Source: MeSH NLM).

\section{INTRODUCCIÓN}

La Federación Internacional de Ginecólogos y Obstetras (FIGO) en el año 1958, definió a la 'edad materna avanzada' como aquella mujer que se embaraza a partir de $\geq 35$ años de edad ${ }^{1-3}$.

El embarazo en mujeres de $\geq 35$ años de edad trae aparejado complicaciones maternas y perinatales por lo que es considerado como alto riesgo obstétrico; y al igual que las menores de 19 años de edad se clasifican como grupos de riesgo en las edades extremas de la vida ${ }^{4}$.

La prevalencia de la edad materna avanzada dentro de todos los embarazos se ha reportado alrededor de 1,5\%; sin embargo, estas cifras pueden variar de acuerdo a la población estudiada ${ }^{5}$.

Las gestantes con edad materna avanzada tienen mayor riesgo de aborto espontáneo, anomalías cromosómicas, malformaciones congénitas, diabetes gestacional, amenaza de parto prematuro y parto prematuro, hemorragia del primer y tercer trimestre, hemorragia post parto, parto por cesárea, y trastornos hipertensivos del embarazo sobre todo la preeclampsia que trae consigo la restricción de crecimiento intrauterino ${ }^{6-10}$.

En el Instituto Nacional Materno perinatal ${ }^{11}$ durante el periodo 2016 ocurrieron 21,983 nacimientos/año, de los cuales corresponden a edad materna avanzada $\geq 35$ años

\footnotetext{
Médico GínecoObstetra. Magíster en Salud Reproductiva. Profesor de la Facultad de Medicina Humana de la Universidad San Martín de Porres y de Ciencias de la Salud de la Universidad Norbert Wiener. Jefe del Servicio de Climaterio y Menopausia del Instituto Nacional Materno Perinatal. Miembro de la Red Latinoamericana de Investigadores en Climaterio (REDLINC). Lima-Perú.

2 Médico Ginecólogo Obstetra, Director del Instituto Nacional Materno Perinatal. Miembro de American College of Obstetricians and Gynecologists (ACOG). Coordinador de Asistencia Técnica del Instituto de Salud Popular, Consultor de Pathfinder International, Docente de la Universidad Nacional Mayor de San Marcos, Lima, Perú ${ }^{3}$ Médico GínecoObstetra. Ex Residente de Ginecología y Obstetricia de la Universidad Nacional Mayor de San Marcos. Sede Instituto Nacional Materno Perinatal. Ex Director del Hospital II-1 Cangallo, Ayacucho. Lima-Perú.

4 Médico Cirujano. Universidad Privada San Juan Bautista. Lima-Perú.

Médico GínecoObstetra. Asistente del Servicio de Obstetricia C. Instituto Nacional Materno Perinatal. Lima-Perú.

Interno de Medicina de la Facultad de Medicina Humana de la Universidad Nacional Federico Villarreal, Lima-Perú.

Médico GínecoObstetra. Profesor Principal de la Universidad Nacional Mayor de San Marcos. Director Ejecutivo de Investigación, Docencia y Atención en Obstetricia y Ginecología del Instituto Nacional Materno Perinatal. Lima-Perú.

8 Médico GínecoObstetra. Profesor Principal de la Universidad Nacional Mayor de San Marcos. Director Ejecutivo de la Oficina de Investigación y Docencia Especializada. Instituto Nacional Materno Perinatal. Lima-Perú.

9 Licenciada en Trabajo Social. Servicio de Trabajo Social. Instituto Nacional Materno Perinatal. Lima-Perú.
} 
de edad con 4,300 gestantes/año que representan el $19,56 \% ; y \geq 40$ años de edad con 1222 gestantes/año que representan el $4,89 \%$.

En la actualidad debido a cambios culturales, económicos y sociales acontecidos en la sociedad mundial, se observa una tendencia por parte de la mujer a retrasar el embarazo a edades cada vez mayores ${ }^{5,8,12-14}$.

El deseo de la mujer de tener un embarazo después de los 35 e incluso 40 años de edad, se ha convertido por ello en un importante fenómeno social por esta modificación en el patrón reproductivo que conlleva a posibles riesgos tanto para la madre como para el feto ${ }^{5,8,15-18}$.

La edad materna avanzada tiene posibilidad de presentar complicaciones, tales como: trastornos hipertensivos, diabetes gestacional, leiomiomatosis uterina, presentaciones anormales, hemorragia obstétrica y mayor índice de cesáreas; así como, restricción del crecimiento intrauterino, parto pretérmino, bajo peso al nacer, puntuación baja en la escala de Apgar, incremento de anomalías congénitas, aborto y aumento en la morbimortalidad materno ${ }^{5,12,19-26}$.

El objetivo de la presente investigación es identificar la morbilidad obstétrica en gestantes con edad materna avanzada atendidas en el Instituto Nacional Materno Perinatal durante el periodo comprendido desde 1 de enero al 30 de junio 2016 .

\section{MATERIALES Y MÉTODOS}

Investigación observacional, descriptivo y retrospectivo.

Población: Gestantes $\geq 35$ años con edad, nulípara o multípara, que fueron atendidas en INMP, desde 1 de enero al 30 de junio del 2016.

Muestra: El número total de nacimientos durante el periodo 2016 en el Instituto Nacional Materno Perinatal fueron 21983 y el número total de egresos maternos en edad materna avanzada fueron 4300/año. El cálculo del tamaño de la muestra con grado de confianza al 95\% y error $5 \%$ corresponden 321 casos estudiados que fueron seleccionadas utilizando el muestreo aleatorio simple.

\section{Criterios de inclusión}

- Primigesta o multigesta con edad materna avanzada $\geq 35$ años de edad.

- Gestantes con edad gestacional confiable por clínica ó ultrasonografía $\geq$

22 semanas.

- Gestantes que culminaron en parto vaginal o cesarea.

- Historias clínicas de egresos maternos registrados en el Instituto Nacional

Materno Perinatal durante el periodo de 01 de enero al 30 de junio 2016 .

\section{Criterios de exclusión}

- Todas las pacientes que no cumplan los criterios de inclusión.

- Gestantes menores de 35 años de edad.

- Gestantes atendidas fuera del Instituto.

Técnicas de recolección de datos: Los datos fueron obtenidos a partir de las historias clínicas de las gestantes atendidas en el Departamento de Obstetricia y Perinatología del INMP cuyo egreso materno corresponde al primer semestre 2016, que fueron consignados en la ficha de recolección de datos, elaborada para dicho propósito.

Técnicas para el procesamiento de la información: Se elaboró una base de datos en el programa Microsoft Windows Excel. Para el procesamiento de la información se utilizó el paquete estadístico SPSS - PASW versión 20 (Stadistical Packaged for the Social Sciences - Predictive Analytics Software) para la elaboración de tablas descriptivas, de frecuencia, así como también de gráficos.

Aspectos éticos: Todos los procedimientos del presente estudio tratan de preservar la integridad y los derechos fundamentales de los pacientes sujetos a investigación, de acuerdo con los lineamientos de las buenas prácticas clínicas y de ética en investigación biomédica. Se garantizó la confidencialidad de los datos obtenidos.

\section{RESULTADOS}

En el Instituto Nacional Materno perinatal durante el periodo 2016 ocurrieron 21,983 nacimientos/año, de los cuales corresponden a edad materna avanzada $\geq 35$ años de edad con 4,300 gestantes/año que representan el $19,56 \% ; y \geq 40$ años de edad con 1222 gestantes/año que representan el $4,89 \%$.

Las características sociodemográficas de 321 gestantes en edad materna avanzada que participaron en el estudio se describen en la tabla 1 . Se aprecia que la edad promedio de las gestantes es $38,8 \pm 3,4$ años, donde la mayoría pertenece al grupo etario de 35 a 39 años con 60,4\%(194 casos) y $6,6 \%$ presentan entre $45-49$ años de edad (21 casos). En relación al estado conyugal corresponden en la mayoría en $63,9 \%$ (205 casos) a gestantes convivientes. Respecto al nivel de escolaridad, el 68,3\% (218 casos) tienen secundaria completa. En cuanto a la ocupación la mayoría son ama de casa con el $80,4 \%$ (255 casos) y predomina el nivel socio económico bajo con el $66,5 \%$ (212 casos) de las gestantes.

Según se aprecia en la tabla 2 en relación al número de control prenatal el $31 \%$ (98 casos) no tuvieron control prenatal que corresponde a la tercera parte de la casuística. Asimismo el 35\% (110 casos) tuvieron adecuado control prenatal que supera los 7 a más controles prenatales. La mayoría de casos tuvieron control prenatal adecuado 
Tabla 1. Características sociodemográficas en edad materna avanzada. Instituto Nacional Materno Perinatal, enero-junio 2016.

\begin{tabular}{|c|c|c|}
\hline $\begin{array}{c}\text { Características } \\
\text { Sociodemográficas }\end{array}$ & \multicolumn{2}{|c|}{ X \pm DS (min. - máx.) } \\
\hline \multirow{2}{*}{ Edad } & \multicolumn{2}{|c|}{$38,8 \pm 3,4(35-47)$} \\
\hline & $\mathbf{N}$ & $\%$ \\
\hline 35 - 39 años & 194 & $60,4 \%$ \\
\hline 40 - 44 años & 106 & $33,0 \%$ \\
\hline 45 - 49 años & 21 & $6,6 \%$ \\
\hline \multicolumn{3}{|l|}{ Estado Civil } \\
\hline Soltera & 25 & $7,8 \%$ \\
\hline Conviviente & 205 & $63,9 \%$ \\
\hline Casada & 91 & $28,3 \%$ \\
\hline \multicolumn{3}{|l|}{ Escolaridad } \\
\hline Sin instrucción & 1 & $0,3 \%$ \\
\hline Primaria & 36 & $11,3 \%$ \\
\hline Secundaria completa & 218 & $68,3 \%$ \\
\hline Superior técnico & 35 & $11,0 \%$ \\
\hline Superior universitario & 29 & $9,1 \%$ \\
\hline \multicolumn{3}{|l|}{ Ocupación } \\
\hline Ama de casa & 255 & $80,4 \%$ \\
\hline Empleada & 29 & $9,1 \%$ \\
\hline Obrera & 7 & $2,2 \%$ \\
\hline Independiente & 26 & $8,2 \%$ \\
\hline \multicolumn{3}{|l|}{ Nivel socioeconómico } \\
\hline Bajo & 212 & $66,5 \%$ \\
\hline Medio & 103 & $32,3 \%$ \\
\hline Alto & 4 & $1,3 \%$ \\
\hline TOTAL (n) & 321 & $100,00 \%$ \\
\hline
\end{tabular}

Tabla 2. Control prenatal versus edad materna avanzada. Instituto Nacional Materno Perinatal. Enero-junio 2016

\begin{tabular}{|c|c|c|c|c|c|c|}
\hline \multirow{3}{*}{ Edad materna } & \multicolumn{6}{|c|}{ Número de controles prenatales } \\
\hline & \multicolumn{2}{|c|}{ De 0 -3 CPN } & \multicolumn{2}{|c|}{ De 4 - 6 CPN } & \multicolumn{2}{|c|}{ De 7 a más CPN } \\
\hline & $\mathbf{N}$ & $\%$ & $\mathbf{N}$ & $\%$ & $\mathbf{N}$ & $\%$ \\
\hline $35-39$ años & 58 & $18,3 \%$ & 66 & $21,3 \%$ & 64 & $20,4 \%$ \\
\hline $40-44$ años & 33 & $10,5 \%$ & 33 & $10,7 \%$ & 38 & $12,1 \%$ \\
\hline 45 - 49 años & 7 & $2,2 \%$ & 6 & $2,0 \%$ & 8 & $2,5 \%$ \\
\hline Total & 98 & $31,0 \%$ & 105 & $34,0 \%$ & 110 & $35,0 \%$ \\
\hline
\end{tabular}

entre las edades de 35 a 39 años y presentaron escaso control prenatal a partir de los 45 a 49 años de edad.

Tabla 3. Antecedentes patológicos según edad materna avanzada. Instituto Nacional Materno Perinatal. Enerojunio 2016

\begin{tabular}{|c|c|c|c|c|c|c|c|c|c|c|}
\hline \multirow{3}{*}{$\begin{array}{l}\text { Edad } \\
\text { materna } \\
\text { (Años) }\end{array}$} & \multicolumn{10}{|c|}{ Antecedentes patológicos } \\
\hline & \multicolumn{2}{|c|}{$\begin{array}{l}\text { Hipertensión } \\
\text { crónica }\end{array}$} & \multicolumn{2}{|c|}{$\begin{array}{l}\text { Diabetes } \\
\text { mellitus }\end{array}$} & \multicolumn{2}{|c|}{ Miomas } & \multicolumn{2}{|c|}{ Otros } & \multicolumn{2}{|c|}{ Ninguno } \\
\hline & $\mathbf{N}$ & $\%$ & $\mathbf{N}$ & $\%$ & $\mathbf{N}$ & $\%$ & $\mathbf{N}$ & $\%$ & $\mathbf{N}$ & $\%$ \\
\hline $35-39$ & 5 & $1,5 \%$ & 6 & $1,9 \%$ & 6 & $1,8 \%$ & 16 & $5,3 \%$ & 150 & $49,20 \%$ \\
\hline $40-44$ & 3 & $0,9 \%$ & 4 & $1,2 \%$ & 6 & $1,8 \%$ & 5 & $1,7 \%$ & 85 & $27,9 \%$ \\
\hline $45-49$ & 2 & $0,6 \%$ & 3 & $0,9 \%$ & 1 & $0,4 \%$ & 0 & $0,0 \%$ & 15 & $4,9 \%$ \\
\hline Total & 10 & $3,0 \%$ & 13 & $4,0 \%$ & 13 & $4,0 \%$ & 21 & $7,0 \%$ & 250 & $82,0 \%$ \\
\hline
\end{tabular}

En relación a los antecedentes patológicos, se aprecia en la tabla 3, que la mayoría de los casos $82 \%$ (250 casos) no presentaron ningún antecedente patológico de importancia. Cabe precisar que solo una mínima parte de la casuística de $3,0 \%, 4,0 \%$ y $4,0 \%$ presentaron hipertensión crónica, diabetes mellitus y miomatosis uterina respectivamente.

Tabla 4. Paridad y edad materna avanzada. Instituto Nacional Materno Perinatal. Enero-junio 2016

\begin{tabular}{|c|c|c|c|c|c|c|c|c|c|}
\hline \multirow{3}{*}{$\begin{array}{c}\text { Edad } \\
\text { materna }\end{array}$} & \multicolumn{8}{|c|}{ Paridad } & \multirow{3}{*}{$\mathbf{p}^{*}$} \\
\hline & \multicolumn{2}{|c|}{ Nulípara } & \multicolumn{2}{|c|}{ Primípara } & \multicolumn{2}{|c|}{ Multípara } & \multicolumn{2}{|c|}{$\begin{array}{c}\text { Gran } \\
\text { multípara } \\
\geq 6\end{array}$} & \\
\hline & $\mathbf{N}$ & $\%$ & $\mathbf{N}$ & $\%$ & $\mathbf{N}$ & $\%$ & $\mathbf{N}$ & $\%$ & \\
\hline 35 - 39 años & 11 & $3,7 \%$ & 23 & $7,66 \%$ & 145 & $48,0 \%$ & 5 & $1,5 \%$ & \\
\hline 40 - 44 años & 3 & $1,0 \%$ & 10 & $3,33 \%$ & 73 & $24,1 \%$ & 14 & $4,3 \%$ & 0,006 \\
\hline 45 - 49 años & 1 & $0,3 \%$ & 3 & $1,0 \%$ & 12 & $3,9 \%$ & 4 & $1,2 \%$ & \\
\hline Total & 15 & $5,0 \%$ & 36 & $12,0 \%$ & 230 & $76,0 \%$ & 23 & $7,0 \%$ & \\
\hline
\end{tabular}

Según se aprecia en la tabla 4 corresponden a la mayoría de la casuística con $76 \%$ (230 casos) a multíparas, seguida de primípara con $12 \%$ (36 casos) y 7,0\% (23 casos) corresponden a gran multípara. De todos ellos corresponden a grandes multíparas en la mayoría entre 40 a 44 años de edad y a primíparas entre las edades 35 a 39 años de edad.

Tabla 5. Edad gestacional según edad materna avanzada. Instituto Nacional Materno Perinatal. Enero-junio 2016.

\begin{tabular}{|c|c|c|c|c|c|c|c|c|c|}
\hline \multirow{3}{*}{$\begin{array}{c}\text { Edad } \\
\text { materna } \\
\text { avanzada }\end{array}$} & \multicolumn{8}{|c|}{$\begin{array}{l}\text { EDAD GESTACIONAL } \\
\text { (semanas) }\end{array}$} & \multirow{3}{*}{$\mathbf{p}^{*}$} \\
\hline & \multicolumn{2}{|c|}{22 a 27} & \multicolumn{2}{|c|}{28 a 36} & \multicolumn{2}{|c|}{37 a 41} & \multicolumn{2}{|c|}{42 a más } & \\
\hline & $\mathbf{N}$ & $\%$ & $\mathbf{N}$ & $\%$ & $\mathbf{N}$ & $\%$ & $\mathbf{N}$ & $\%$ & \\
\hline 35 - 39 años & 5 & $2,0 \%$ & 31 & $10,0 \%$ & 151 & $48,7 \%$ & 2 & $0,7 \%$ & \\
\hline 40 - 44 años & 0 & $0,0 \%$ & 24 & $7,7 \%$ & 75 & $24,2 \%$ & 0 & $0,0 \%$ & 0,077 \\
\hline 45 - 49 años & 0 & $0,0 \%$ & 7 & $2,3 \%$ & 13 & $4,1 \%$ & 1 & $0,3 \%$ & \\
\hline Total & 5 & $2,0 \%$ & 62 & $20,0 \%$ & 239 & $77,0 \%$ & 3 & $1,0 \%$ & \\
\hline
\end{tabular}

En relación a la edad gestacional, según se aprecia la tabla 5 , corresponden a pretérmino $\leq 36$ semanas $22,0 \%$ ( 67 casos) y solamente el $1 \%$ ( 3 casos) a > 42 semanas. La mayoría estuvieron ubicados en embarazos a término en $77 \%$ (239 casos).

Tabla 6. Vía de culminación del parto según edad materna avanzada. Instituto Nacional Materno Perinatal. Enero-junio 2016

\begin{tabular}{ccccc}
\hline \multirow{2}{*}{$\begin{array}{l}\text { Edad materna } \\
\text { avanzada }\end{array}$} & \multicolumn{2}{c}{ Vaginal } & \multicolumn{2}{c}{ Cesárea } \\
\cline { 2 - 5 } & $\mathbf{N}$ & $\mathbf{\%}$ & $\mathbf{N}$ & $\mathbf{\%}$ \\
\cline { 2 - 5 } & 87 & $27,1 \%$ & 109 & $33,9 \%$ \\
\hline $35-39$ años & 44 & $13,8 \%$ & 60 & $18,7 \%$ \\
$40-44$ años & 10 & $3,1 \%$ & 11 & $3,4 \%$ \\
\hline $45-49$ años & $\mathbf{1 4 1}$ & $\mathbf{4 4 , 0 \%}$ & $\mathbf{1 8 0}$ & $\mathbf{5 6 , 0} \%$ \\
\hline Total & & & &
\end{tabular}

La vía de culminación del parto como se aprecia en la tabla 6 corresponden en la mayoría de la casuística con $56 \%$ (180 casos) a cesárea y de ellas en la mayoría corresponden de 35 a 39 años de edad. La vía de parto 
vaginal ocurrieron en el $44 \%$ (141 casos) y de ellas en la mayoría entre 35 a 39 años de edad.

Tabla 7. Diagnóstico principal de cesárea en edad materna avanzada. Instituto Nacional Materno Perinatal. Enero-junio 2016.

\begin{tabular}{lcccc}
\hline \multirow{2}{*}{ Causa de cesárea } & \multicolumn{2}{c}{ Sí } & \multicolumn{2}{c}{ No } \\
\cline { 2 - 5 } & $\mathbf{N}$ & $\%$ & $\mathbf{N}$ & $\%$ \\
\hline Cesareada anterior & 80 & $24,9 \%$ & 241 & $75,1 \%$ \\
\hline $\begin{array}{l}\text { Posición fetal anómala } \\
\text { (podálica/transversa) }\end{array}$ & 30 & $9,3 \%$ & 291 & $90,7 \%$ \\
\hline Preeclampsia severa & 26 & $8,1 \%$ & 295 & $91,9 \%$ \\
\hline Incompat Cefalo pelv & 25 & $7,8 \%$ & 296 & $92,2 \%$ \\
\hline Sufrimiento fetal agudo & 20 & $6,2 \%$ & 301 & $93,8 \%$ \\
\hline Distocia funicular & 13 & $4,0 \%$ & 308 & $96,0 \%$ \\
\hline RCIU & 12 & $3,7 \%$ & 309 & $96,3 \%$ \\
\hline Embarazo múltiple & 10 & $3,1 \%$ & 311 & $96,9 \%$ \\
\hline Placenta previa & 7 & $2,2 \%$ & 314 & $97,8 \%$ \\
\hline RPM prolongado & 5 & $1,6 \%$ & 316 & $98,4 \%$ \\
\hline DPP & 3 & $0,9 \%$ & 318 & $99,1 \%$ \\
\hline
\end{tabular}

Como se aprecia en la tabla 7 , la causa principal de la cesárea fue en la mayoría de ellas en el $24,9 \%$ (80 casos) por motivo de cesárea previa de 1 a más veces. La segunda causa de cesárea corresponde en el 9,3\% (30 casos) a posición fetal anómala como podálica y/o transversa. Cabe destacar la tercera causa de cesárea corresponde a preeclampsia severa en $8,1 \%$ (26 casos); seguidamente incompatibilidad cefalopélvica en $7,8 \%$ (25 casos); sufrimiento fetal agudo en 6,2\% (20 casos); distocia funicular en $4 \%$ (13 casos) y RCIU en $3,7 \%$ (12 casos).

En la tabla 8 se aprecian que las morbilidades obstétricas encontradas fueron la preeclampsia en $11,3 \%$ en los grupos etarios de 40-44 años y 19\% entre 45-49 años de edad. Cabe destacar que el parto pretérmino alcanzó significancia estadística $(p<0,001)$ en todos los grupos etarios con $10,3 \%, 9,4 \%$ y $38,1 \%$ entre los grupos etarios de 35-39 años, 40-44 años, y 45-49 años de edad respectivamente. Por otro lado, la ruptura prematura de

Tabla 8. Morbilidad obstétrica y edad materna avanzada. Instituto Nacional Materno Perinatal. Enero-junio 2016

\begin{tabular}{|c|c|c|c|c|c|c|c|}
\hline \multirow{3}{*}{ Morbilidad obstétrica } & \multicolumn{6}{|c|}{ Edad materna avanzada } & \multirow{3}{*}{$\mathbf{p}$} \\
\hline & \multicolumn{2}{|c|}{35 - 39 años } & \multicolumn{2}{|c|}{40 - 44 años } & \multicolumn{2}{|c|}{45 - 49 años } & \\
\hline & $\mathrm{N}=194$ & $\%$ & $\mathrm{~N}=106$ & $\%$ & $\mathrm{~N}=\mathbf{2 1}$ & $\%$ & \\
\hline \multicolumn{8}{|l|}{ Diabetes gestacional } \\
\hline Sí & 2 & $1,0 \%$ & 4 & $3,8 \%$ & 1 & $4,8 \%$ & 0,21 \\
\hline No & 192 & $99,0 \%$ & 102 & $96,2 \%$ & 20 & $95,2 \%$ & \\
\hline \multicolumn{8}{|l|}{ Hipertensión gestacional } \\
\hline Sí & 5 & $2,6 \%$ & 7 & $6,6 \%$ & 1 & $4,8 \%$ & 0,236 \\
\hline No & 189 & $97,4 \%$ & 99 & $93,4 \%$ & 20 & $95,2 \%$ & \\
\hline \multicolumn{8}{|l|}{ Preeclampsia } \\
\hline Sí & 17 & $8,8 \%$ & 12 & $11,3 \%$ & 4 & $19,0 \%$ & 0,307 \\
\hline No & 177 & $91,2 \%$ & 94 & $88,7 \%$ & 17 & $81,0 \%$ & \\
\hline \multicolumn{8}{|l|}{$\begin{array}{l}\text { Ruptura Prematura } \\
\text { Membranas }\end{array}$} \\
\hline Sí & 26 & $13,4 \%$ & 19 & $17,9 \%$ & 3 & $14,3 \%$ & 0,574 \\
\hline No & 168 & $86,6 \%$ & 87 & $82,1 \%$ & 18 & $85,7 \%$ & \\
\hline \multicolumn{8}{|l|}{ Parto pretérmino } \\
\hline Sí & 20 & $10,3 \%$ & 10 & $9,4 \%$ & 8 & $38,1 \%$ & 0,001 \\
\hline No & 174 & $89,7 \%$ & 96 & $90,6 \%$ & 13 & $61,9 \%$ & \\
\hline \multicolumn{8}{|c|}{ Alumbramiento incompleto } \\
\hline Sí & 8 & $4,1 \%$ & 4 & $3,8 \%$ & 1 & $4,8 \%$ & 0,975 \\
\hline No & 186 & $95,9 \%$ & 102 & $96,2 \%$ & 20 & $95,2 \%$ & \\
\hline \multicolumn{8}{|l|}{ Atonía/Hipotonía uterina } \\
\hline Sí & 13 & $6,7 \%$ & 6 & $5,7 \%$ & 1 & $4,8 \%$ & 0,9 \\
\hline No & 181 & $93,3 \%$ & 100 & $94,3 \%$ & 20 & $95,2 \%$ & \\
\hline \multicolumn{8}{|l|}{ Desgarro Perineal I grado } \\
\hline Sí & 36 & $18,6 \%$ & 20 & $18,9 \%$ & 2 & $9,5 \%$ & 0,573 \\
\hline No & 158 & $81,4 \%$ & 86 & $81,1 \%$ & 19 & $90,5 \%$ & \\
\hline \multicolumn{8}{|c|}{ Anemia moderada o severa } \\
\hline Sí & 36 & $18,6 \%$ & 14 & $13,2 \%$ & 2 & $9,5 \%$ & 0,336 \\
\hline No & 158 & $81,4 \%$ & 92 & $86,8 \%$ & 19 & $90,5 \%$ & \\
\hline \multicolumn{8}{|l|}{ Infección urinaria } \\
\hline Sí & 7 & $3,6 \%$ & 3 & $2,8 \%$ & 2 & $9,5 \%$ & 0,332 \\
\hline No & 187 & $96,4 \%$ & 103 & $97,2 \%$ & 19 & $90,5 \%$ & \\
\hline
\end{tabular}


membranas se encontró en el $13,4 \%, 17,9 \%$ y $14,3 \%$ entre los grupos etarios 35-39 años, 40-44 años y 45-49 años respectivamente. Se hallaron desgarro perineal I grado en el $18,6 \%, 18,9 \%$ y $9,5 \%$ entre el grupo etario de 35-39 años, 40-44 y 45-49 años respectivamente. La anemia moderada/severa se encontró en el 18,6\%, 13,2\% y $9,5 \%$ entre los grupos etarios de 35-39 años, $40-44$ y 45 49 años respectivamente. Finalmente, infección urinaria se halló en $3,6 \%, 2,8 \%$ y 9,5\% entre los grupos etarios de 35-39 años, 40-44 y 45-49 años respectivamente.

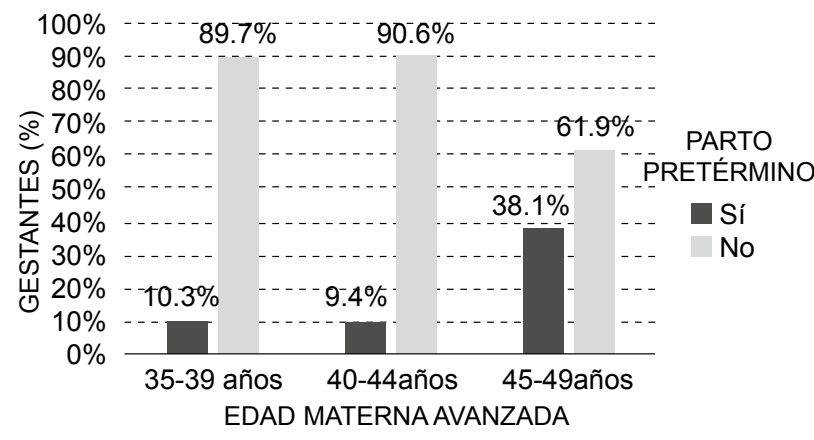

Gráfico 1. Parto pretérmino como complicación obstétrica según edad materna avanzada. Instituto Nacional Materno perinatal. Enero-junio 2016.

Como se aprecia en el gráfico 1, el parto pretérmino, como factor de riesgo obstétrico, alcanzó significancia estadística $(p<0,001)$ entre los grupos etarios de 35-39 años, 40-44 años, y 45-49 años de edad con 10,3\%, 9,4\% y $38,1 \%$ respectivamente. El mayor porcentaje de parto pretérmino ocurrió en el grupo etario entre 45-49 años de edad.

Tabla 9. Causas de muerte materna y razón de muerte materna (RMM) en el Instituto Nacional Materno PerinatalI semestre 2016

\begin{tabular}{lccc}
\hline $\begin{array}{l}\text { CAUSA DE MUERTE } \\
\text { MATERNA }\end{array}$ & Total & $\%$ & $\begin{array}{c}\text { Razón de Muerte } \\
\text { Materna } \\
\text { (N MM/Total }\end{array}$ \\
\hline $\begin{array}{l}\text { Sepsis e infección } \\
\text { puerperal }\end{array}$ & 2 & 66,7 & 9,14 \\
Hemorragia postparto & 1 & 33,3 & 4,56 \\
TOTAL & 3 & 100 & 13,70 \\
\hline
\end{tabular}

Como se aprecia en la tabla 9, en el Instituto Nacional Materno Perinatal durante el I semestre 2016 se registraron 3 muertes maternas y ocurrieron 21,893 nacimientos/año; por lo que la RMM calculada entre número de muertes maternas/total de nacimientos al año multiplicado por cien mil arroja 13,70 defunciones por cada 100 mil nacidos vivos.

Como se aprecia en la tabla 10 en relación a muerte materna en edad materna avanzada durante el I semestre 2016 se registró solo un caso de muerte materna; por lo que, la razón de muerte materna, arroja 4,56 x 100 000; y la principal causa de defunción materna fue la hemorragia postparto.
Tabla 10. Causas de mortalidad materna en gestantes con edad materna avanzada en el Instituto Nacional Materno Perinatal- I semestre 2016

\begin{tabular}{|c|c|c|c|c|}
\hline FILIACIÓN & $\begin{array}{l}\text { EDAD } \\
\text { (años) }\end{array}$ & $\begin{array}{c}\text { CAUSA DE } \\
\text { MUERTE } \\
\text { MATERNA }\end{array}$ & $\begin{array}{c}\text { ESTANCIA } \\
\text { HOSPITALARIA }\end{array}$ & $\begin{array}{c}\text { RAZON DE } \\
\text { MUERTE } \\
\text { MATERNA } \\
\text { (No MM/Total } \\
\text { nacimientos } \\
\text { x 100000) }\end{array}$ \\
\hline S.E.Z & 41 & $\begin{array}{c}\text { Hemorragia } \\
\text { postparto }\end{array}$ & 11horas & $\begin{array}{c}4,56 \times \\
100,000 \\
\end{array}$ \\
\hline
\end{tabular}

\section{DISCUSIÓN}

La morbilidad obstétrica continúa siendo un problema de salud pública, en especial en gestantes en edad materna avanzada ${ }^{14,27}$, las cuales concuerdan con recientes investigaciones de autores como Hurtado $\mathrm{CJ}^{28}$ y Gutiérrez EC ${ }^{29}$ quienes comunican que en las edades extremas de la vida se presentan altas tasas de complicaciones obstétricas y perinatales.

En relación a factores de riesgo obstétricos en nuestra casuística el $31 \%$ no tuvieron control prenatal y escaso control prenatal a partir de los 45 a 49 años de edad; asimismo, el $17 \%$ fueron nulíparas o primíparas; las cuales difieren de las publicaciones de Baranda-Nájera $\mathrm{N}$ y $\mathrm{col}^{30}$ donde la nuliparidad alcanzó un $42,47 \%$. Al respecto, Obregón LE ${ }^{31}$ menciona que la nuliparidad $a \geq 36$ años de edad aumentan 3,3 veces el riesgo de complicaciones materno-fetales, tales como las anormalidades congénitas, abortos, óbitos, diabetes, macrosomía, enfermedad hipertensiva del embarazo, menor calificación en la escala de Apgar y anormalidades en la inserción placentaria.

Según Nolasco-Blé AK ${ }^{17}$ la vía más frecuente de terminación del embarazo fue la cesárea y la principal causa de indicación de cesárea es arbitrariamente el antecedente de cesárea. Estudios de Lemor A 32 reportan que la edad materna avanzada se asoció con una mayor tasa de cesáreas y más riesgo de tener un hijo con síndrome de Down; Ventura $W$ y col ${ }^{33}$ reporta alta tasa de cesáreas $(56,8 \%)$ y mayor en el grupo de nulíparas (71,7\%; $\mathrm{p}<0,01)$; Baranda-Nájera $\mathrm{N}$ y col 30 reportan prevalencia de cesárea en $61,18 \%$; todas ellas concuerdan con nuestra casuística donde la vía de culminación del parto predominante fue la cesárea en $56 \%$ de los casos, y las tres principales causas fueron: cesárea previa, posición fetal anómala como podálica y/o transversa y preeclampsia severa. Asimismo, CurielBalsera $\mathrm{E}$ et al ${ }^{34}$ observaron que una indicación frecuente para realizar cesárea en mujeres $\geq 40$ años es el trabajo de parto en fase latente prolongado.

Estudios de Olórtegui LF ${ }^{35}$, mencionan que las complicaciones obstétricas frecuentes en gestantes con edad materna avanzada son la rotura prematura de membranas, hipodinamia uterina y el alumbramiento 
incompleto; y los factores que se asociaron significativamente fueron alto índice de masa corporal, obesidad, cesárea previa y paridad. Asimismo, estudios de Hurtado CJ ${ }^{28}$ reportan como complicaciones obstétricas en las gestantes añosas: anemia $(24,5 \%)$, ITU $(23,5 \%)$, desgarro perineal $(14,7 \%)$, APP $(10,8 \%)$ y pre eclampsia $(8,8 \%)$; y Nolasco-Blé AK ${ }^{17}$, informa que la complicación materna más frecuente fue la diabetes gestacional $(16 \%)$ seguida de los trastornos hipertensivos del embarazo (10\%). Por otro lado, Gutierrez E ${ }^{29}$ mencionan complicaciones obstétricas como: preeclampsia y ruptura prematura de membranas, parto pretérmino, desproporción céfalo-pélvica , desgarros perineales, oligohidramnios, desprendimiento prematuro de placenta, placenta previa y atonía uterina, diabetes gestacional, polihidramios y acretismo placentario; y finalmente PeñaAyudante $\mathrm{R}$ y $\mathrm{col}^{36}$ reportan que las primigestas añosas tuvieron con más frecuencia hipertensión inducida por el embarazo, hemorragia del primer trimestre, embarazo múltiple; todas ellas coinciden con los hallazgos de nuestra casuística donde predominan las complicaciones obstétricas como: preeclampsia; ruptura prematura de membranas; desgarro perineal I grado; anemia moderada/ severa e infección urinaria; y el parto pretérmino que alcanzó significancia estadística $(p<0,001)$. Recientes investigaciones publicadas por Baranda-Nájera $\mathrm{N}$ y col ${ }^{30}$ en edad materna avanzada y morbilidad obstétrica reportan que las principales comorbilidades que se presentaron en el segundo trimestre de la gestación fueron diabetes gestacional $(41,37 \%)$, ruptura prematura de membranas $(37,96 \%)$ e hipertensión gestacional $(20,61 \%)$. En el tercer trimestre correspondió a placenta previa $(30,0 \%)$, oligohidramnios severo $(50,0 \%)$, preeclampsia severa $(10,0 \%)$ y trombosis venosa profunda $(10,0 \%)$.

En relación a la razón de muerte materna en edad materna avanzada en nuestra casuística arroja 4,56 x 100 000 nacimientos y la principal causa de defunción fue la hemorragia postparto.

Se puede concluir que las principales morbilidades obstétricas en EMA son parto pretérmino, preeclampsia, ruptura prematura de membranas, anemia moderada/ severa y alta tasa de cesárea.

\section{REFERENCIAS BIBLIOGRAFICAS}

1. Huang L, Sauve R, Birkett N, Fergusson D, van Walraven C. Maternal age and risk of stillbirth: a systematic review. CMAJ 2008; 178 (2): 165-72.

2. Ataulla I. The older obstetric patient. Current Obstet Gynaecol. 2005; 15:46-53.

3. Hamilton BE, Martin JA, Ventura SJ. Births: preliminary data from 2007. Natl Vital Stat Rep 2009; 57:1-23.

4. Sánchez García M, Muñoz Silva A. Influencia de padres y amigos sobre la actitud hacia la conducta sexual de prevención en la adolescencia. Un análisis función de género. Rev. Latinoamericana de Psicolog 2005: 37 (1).

5. Başer E, Seçkin KD, Erkıınç $S$, Karslı MF, Yeral IM, Kaymak O, Çağlar T, Danışman N. The impact of parity on perinatal outcomes in pregnancies complicated by advanced maternal age. J Turkish-German Gynecol Assoc 2013; 14: 205-9.

6. Heffner L. Advanced maternal age. How old is too old? N Engl J Med 2004; 351(19):1927-9.

7. Bardin R, Melamed N, Tenebaun-Gavish $\mathrm{K}$, Arbid $\mathrm{N}$, Meyerovitch T, Ben-Haroush A, et al. Pregnancy outcome at extremely advenced maternal age. Am J Obstet Gynecol. 2007; 197(6)Supl 1:80-8.

8. Heras P, Gobernado T, Mora C, Almaraz G. La edad materna como factor de riesgo obstétrico. Resultados perinatales en gestantes de edad avanzada. Prog Obstet Ginecol. 2011;54(11):575-580

9. Yogev Y, Melamed N, Tenenbaum-Gavish K, Bardin R, BenShitrit G, Ben-Haroush A. Pregnancy outcome at extremely advanced maternal age. Am J Obstet Gynecol. 2010;203:558. e1-7

10. Romero-Maldonado S, Quezada-Salazar C, López M, Arroyo L. Efecto de riesgo en el hijo de madre con edad avanzada (estudio de casos y controles). Ginecol Obstet Méx. 2002; 70:295-302.

11. Instituto Nacional Materno Perinatal. Boletín estadístico periodo 2016. Lima Perú. Disponible en: http://www.inmp. gob.pe/institucional/boletines-estadisticos/1422371837

12. Tipiani-Rodríguez O. ¿Es la edad materna avanzada un Factor de riesgo independiente para Complicaciones maternoperinatales?. Rev Per Ginecol Obstet. 2006;52(3):89-99

13. Leridon $\mathrm{H}$. Can assisted reproduction technology compensate for the natural decline in fertility with age?. A model assessment. Hum Reprod. 2004;19:1548-53.

14. Wang Y, Tanbo T, Abyholm T, Henriksen T. The impact of advanced maternal age and parity on obstetric and perinatal outcomes in singleton gestations. Arch Gynecol Obstet 2011; 284:31-37

15. Callaway LK, Lust K, Mclntyre HD. Pregnancy outcomes in women of very advanced maternal age. Aust $\mathrm{N} \mathrm{Z} \mathrm{J} \mathrm{Obstet}$ Gynaecol 2005;45: 12-6.

16. Hollier LM, Leveno KJ, Kelly MA, MCIntire DD, Cunningham FG. Maternal age and malformations in singleton births. Obstet Gynecol 2000;96:701-706.

17. Nolasco-Blé AK, Hernández-Herrera RJ, Ramos-González RM. Hallazgos perinatales de embarazos en edad materna avanzada. Ginecol Obstet Mex 2012;80(4):270-275

18. Torres Fuentes $\mathrm{Cl}$. Resultado perinatal y obstétrico en embarazadas mayores de 35 años en el Hospital Nacional de Maternidad en el periodo de enero a diciembre de 2009. (Tesis de Post grado en Ginecología y Obstetricia). Universidad de El Salvador. 2010 pp 37.

19. Baranda-Nájera N, Patiño-Rodríguez DM, Ramírez-Montiel ML, Rosales-Lucio J, Martínez-Zúñiga M,Prado-Alcázar J, Contreras-Carreto NA. Edad materna avanzada y morbilidad obstétrica. Evid Med Invest Salud 2014; 7 (3): 110-113

20. Curiel-Balsera E, Prieto-Palomino MA, Muñoz Bono J, Ruiz E, Galeas JL, Quesada García G. Análisis de la morbimortalidad materna de las pacientes con preeclampsia grave, eclampsia y síndrome de HELLP que Ingresan a la Unidad de Cuidados Intensivos Ginecoobstétrica. Med Intensiva. 2011; 35 (8): 478-483.

21. Lamminpaa R, Vehvilainen-Julkunen K, Gissler $M$, Heinonen S. Preeclampsia complicated by advanced maternal age: a registrybased study on primiparous women in Finland 1997-2008. BMC Pregnancy and Childbirth. 2012; 12: 47-50.

22. Flores Le-Roux JA, Benaiges Boix D, Botet JP. Diabetes mellitus gestacional: importancia del control glucémico intraparto. Clin Invest Arterioscl. 2012; 11: 1- 
23. Bayrampour $\mathrm{H}$, Heaman M. Advanced maternal age and the risk of cesarean birth: A systematic review. Birth. 2010; 37 : 3-9.

24. Schoen C, Rosen T. Maternal and perinatal risks for women over 44. A review. Maturitas. 2009; 64 (2): 109-113.

25. Marín IR, Pérez G, Álvarez N. Hipertensión arterial y embarazo. Nefro plus. 2011; 4 (2): 21-30.

26. Vambergue A, Fajardy I. Consequences of gestational and pregesta cional diabetes on placental function and birth weight. World J Diabetes. 2011; 2 (11): 196-203.

27. Chan BC, Lao TT. Effect of parity and advanced maternal age on obstetric outcome. Int J Gynaecol Obstet 2008;102:237241

28. Hurtado CJ. Complicaciones obstetricas y perinatales en gestantes añosas y adolescentes atendidas en el hospital Iquitos César Garayar García enero a diciembre 2013. (Tesis de pregrado). Lima, Perú: Facultad de Medicina Humana "Rafael Donayre Rojas" .Universidad de Amazonía Peruana, 2014. 48pp.

29. Gutierrez EC. Complicaciones obstétricas y perinatales en gestantes mayores de 35 años en el Hospital Rezola-Cañete en el año 2014. Tesis de Pregrado. Lima, Peru: Facultad de Obstetricia y Enfermería. Universidad San Martín de Porres, 2015. 46 pp. [Fecha de acceso 12 de enero del 2017] URL Disponible en: http://www.repositorioacademico.usmp.edu. pe/bitstream/usmp/2158/3/gutierrez_e.pdf

[Fecha de acceso 12 de enero del 2017] URL Disponible en: http://dspace.unapiquitos.edu.pe/bitstream/ unapiquitos/147/1/Tesis\%20Parte\%201.pdf
30. Baranda-Nájera N, Patiño-Rodríguez D, Ramírez-Montiel ML, Rosales-Lucio J, Martínez-Zúñiga M, Prado-Alcázar JJ, Contreras-Carreto NA. Edad materna avanzada y morbilidad obstétrica. Evid Med Invest Salud 2014; 7 (3): 110-113.

31. Obregón Yáñez LE. Primigesta de edad avanzada. Rev Obstet Ginecol Venez. 2007; 67 (3): 152-166.

32. Lemor A. Relación entre la edad materna avanzada y la morbilidad neonatal en el Hospital Nacional Arzobispo Loayza. Enero -Octubre del 2012. Horiz Med 2014; 14(2): $22-$ 26)

33. Ventura W y col. Embarazo después de los 40 años: características epidemiológicas. Rev Per Ginecol Obstel 2005;5(1):49-52

34. Curiel-Balsera E, Prieto-Palomino MA, Muñoz Bono J, Ruiz E, Galeas JL, Quesada García G. Análisis de la morbimortalidad materna de las pacientes con preeclampsia grave, eclampsia y síndrome de HELLP que Ingresan a la Unidad de Cuidados Intensivos Ginecoobstétrica. Med Intensiva. 2011; 35 (8): 478-483.

35. Olórtegui LF. Factores asociados a las complicaciones obstétricas en gestantes añosas en el Instituto Nacional Materno Perinatal, enero - junio 2013. (Tesis de Pre Grado). Facultad de Medicina de la Universidad Nacional Mayor de San Marcos. Lima-Perú 2014 pp 52.

36. Peña-Ayudante R, Palacios J, Oscuvilca E, Peña A. El primer embarazo en mujeres mayores de 35 años de edad. Rev Per Ginecol Obstet 2011; 57: 49-53
ORCID iDs

Félix Ayala Peralta

Enrique Guevara Ríos

https://orcid. org/0000-0002-2830-3789

Antonio Lina Figueroa

https://orcid.org/0000-0002-6962-2639

(i) https://orcid.org/0000-0003-1527-8649 